\title{
Homozygote CRIM1 variant is associated with thiopurine-induced neutropenia in leukemic patients with both wildtype NUDT15 and TPMT
}

Yoomi Park ${ }^{1 \dagger}$, Hyery Kim ${ }^{2 \dagger}$, Heewon Seo ${ }^{1,3}$, Jung Yoon Choi ${ }^{4,5}$, Youngeun Ma ${ }^{6}$, Sunmin Yun ${ }^{1}$, Byung-Joo Min ${ }^{1}$, Myung-Eui Seo ${ }^{1}$, Keon Hee Yoo ${ }^{7}$, Hyoung Jin Kang ${ }^{4,5}$, Ho Joon Im $^{2^{*}}$ and Ju Han Kim ${ }^{1,8^{*}}$

\begin{abstract}
Background: NUDT15 and TPMT variants are strong genetic determinants of thiopurine-induced hematological toxicity that results in therapeutic failure in pediatric acute lymphoblastic leukemia (ALL). However, many patients with both wild-type (WT) NUDT15 and TPMT still suffer from thiopurine toxicity and therapeutic failure.

Methods: Whole-exome sequencing was done for discovery $(N=244)$ and replication $(N=76)$ cohorts. Age- and sex-adjusted multiple regression analyses of both WT patients were performed to identify $(p<0.01, N=188$ for discovery) and validate ( $p<0.05, N=52$ for replication) candidate variants for the tolerated last-cycle 6 -mercaptopurine (6-MP) dose intensity percentage (DIP). Both independent and additive effects of the candidate variants on wellknown NUDT15 and TPMT were evaluated by multigene prediction models.

Results: Among the 12 candidate variants from the discovery phase, the rs3821169 variant of the gene encoding Cysteine-Rich Transmembrane BMP Regulator 1 (CRIM1) was successfully replicated $(p<0.05)$. It showed high interethnic variability with an impressively high allele frequency in East Asians $(T=0.255)$ compared to Africans $(0.001)$, Americans (0.02), Europeans (0.009), and South Asians (0.05). Homozygote carriers of the CRIM1 rs3821169 variant ( $N=12$, $5 \%$ ) showed significantly lower last-cycle 6-MP DIPs in the discovery, replication, and combined cohorts $(p=0.025$, 0.013, and 0.001, respectively). The traditional two-gene model (NUDT15 and TPMT) for predicting 6-MP DIP <25\% was outperformed by the three-gene model that included CRIM1, in terms of the area under the receiver operating characteristic curve $(0.734$ vs. 0.665$)$, prediction accuracy $(0.759$ vs. 0.756$)$, sensitivity ( 0.636 vs. 0.523$)$, positive predictive value (0.315 vs. 0.288$)$, and negative predictive value (0.931 vs. 0.913 ).
\end{abstract}

Conclusions: The CRIM1 rs3821169 variant is suggested to be an independent and/or additive genetic determinant of thiopurine toxicity beyond NUDT15 and TPMT in pediatric ALL.

Keywords: CRIM1, NUDT15, TPMT, 6-Mercaptopurine, Toxicity, Acute lymphoblastic leukemia

\footnotetext{
*Correspondence: hojim@amc.seoul.kr; juhan@snu.ac.kr

${ }^{\dagger}$ Yoomi Park and Hyery Kim contributed equally to this work

1 Division of Biomedical Informatics, Seoul National University Biomedical

Informatics (SNUBI), Seoul National University College of Medicine, 101

Daehak-ro, Jongno-gu, Seoul 03080, South Korea

2 Department of Pediatrics, Asan Medical Center Children's Hospital,

University of Ulsan College of Medicine, 88, Olympic-ro 43-gil, Songpa-gu,

Seoul 05505, South Korea

Full list of author information is available at the end of the article
}

(C) The Author(s) 2020. This article is licensed under a Creative Commons Attribution 4.0 International License, which permits use, sharing, adaptation, distribution and reproduction in any medium or format, as long as you give appropriate credit to the original author(s) and the source, provide a link to the Creative Commons licence, and indicate if changes were made. The images or other third party material in this article are included in the article's Creative Commons licence, unless indicated otherwise in a credit line to the material. If material is not included in the article's Creative Commons licence and your intended use is not permitted by statutory regulation or exceeds the permitted use, you will need to obtain permission directly from the copyright holder. To view a copy of this licence, visit http://creativeco mmons.org/licenses/by/4.0/. The Creative Commons Public Domain Dedication waiver (http://creativecommons.org/publicdomain/ zero/1.0/) applies to the data made available in this article, unless otherwise stated in a credit line to the data. 


\section{Background}

The associations of NUDT15 and TPMT genetic variants with 6-mercaptopurine (6-MP) intolerance have been very well established in pediatric acute lymphoblastic leukemia (ALL). In European populations, about 50\% of thiopurine-induced severe cytotoxic adverse reactions such as severe neutropenia and leukopenia are explained by NUDT15 and TPMT genetic variants [1]. The Clinical Pharmacogenetics Implementation Consortium (CPIC) [2] publishes practical evidence-based guidelines for the clinical implications of 6-MP based on these two genes, supporting the implementation of pharmacogenetic testing in routine clinical practice $[3,4]$.

Currently, 6-MP dose is clinically titrated based on the known risk variants of TPMT or NUDT15. However, a substantial proportion of leukemia patients who have no genetic variation in NUDT15 or TPMT still suffer from life-threatening toxicity, which may result in dose reduction and/or discontinuation of 6-MP and resultant therapeutic failure and relapse. Therefore, further discovery of novel genetic variants other than NUDT15 and TPMT variations is urgently needed for preventing 6-MP toxicity and improving pediatric ALL patient care.

The present study aimed to identify novel genetic variations associated with the 6-MP intolerance in pediatric ALL patients who carry both wild-type (WT) NUDT15 and TPMT by using whole-exome sequencing (WES) technology. We identified and systematically evaluated the deterministic effects of novel candidate variants on a clinically important hematological toxicity indicator: the last-cycle 6-MP dose intensity percentage (DIP) tolerated by pediatric ALL patients.

\section{Methods}

\section{Subjects}

A total of 320 Korean pediatric ALL patients receiving 6-MP treatment during maintenance therapy include discovery $(N=244)$ and replication $(N=76)$ cohorts, recruited from two teaching hospitals [Seoul National University Hospital (SNUH) and Asan Medical Center (AMC)] and three teaching hospitals [SNUH, AMC, and Samsung Medical Center (SMC)] located in Seoul, Korea, respectively (Table 1 ). The discovery cohort was retrospectively collected and sequenced before February 2018, while the replication cohort was subsequently collected and sequenced from October 2018 to November 2019. All of the selected individuals conformed with the exclusion criteria (i.e., relapse of the disease, stem cell transplantation, Burkitt's lymphoma, mixed phenotype acute leukemia, infant ALL, or very high risk of ALL). The hematological toxicity was estimated based on the measurement of the tolerated last-cycle 6-MP DIP as the
Table 1 Clinical characteristics of pediatric acute lymphoblastic leukemia (ALL) subjects who are normal metabolizers (NMs) for both NUDT15 and TPMT

\begin{tabular}{|c|c|c|c|}
\hline Characteristic & Discovery & Replication & Combined \\
\hline $\begin{array}{c}\text { Number of } \\
\text { subjects }\end{array}$ & 188 & 52 & 240 \\
\hline Age, years ${ }^{a}$ & $6.9 \pm 4.5$ & $7.4 \pm 4.5$ & $7.0 \pm 4.5$ \\
\hline \multicolumn{4}{|l|}{ Sex } \\
\hline Male & 108 & 29 & 137 \\
\hline Female & 80 & 23 & 103 \\
\hline \multicolumn{4}{|c|}{ Last-cycle 6-MP dose, mg/m²/day } \\
\hline$\leq 10$ & $8.68 \pm 1.5(3)$ & $6.29 \pm 2.2(5)$ & $7.19 \pm 2.2(8)$ \\
\hline$>10$ and $\leq 15$ & $13.89 \pm N A(1)$ & $13.21 \pm 1.8(3)$ & $13.38 \pm 1.5(4)$ \\
\hline$>15$ and $\leq 25$ & $18.52 \pm 3.4(5)$ & $22.13 \pm 1.3(4)$ & $20.12 \pm 3.1(9)$ \\
\hline$>25$ and $\leq 35$ & $29.95 \pm 3.3(16)$ & $30.49 \pm 0.8(4)$ & $30.06 \pm 3.0(20)$ \\
\hline$>35$ and $\leq 45$ & $39.54 \pm 3.6(8)$ & $40.18 \pm 2.6(5)$ & $39.79 \pm 3.1(13)$ \\
\hline$>45$ and $\leq 60$ & $52.71 \pm 4.0(41)$ & $54.84 \pm 2.8(5)$ & $52.94 \pm 3.9(46)$ \\
\hline$>60$ and $\leq 80$ & $70.79 \pm 6.0(55)$ & $69.35 \pm 5.1(10)$ & $70.57 \pm 5.8(65)$ \\
\hline$>80$ and $\leq 100$ & $90.87 \pm 5.9(35)$ & $85.98 \pm 5.0(8)$ & $89.96 \pm 6.0(43)^{*}$ \\
\hline$>100$ & $\begin{array}{l}112.67 \pm 16.6 \\
(24)\end{array}$ & $122.66 \pm 23.3(8)$ & $115.17 \pm 18.7(32)$ \\
\hline Total & $\begin{array}{l}68.44 \pm 27.6 \\
\quad(188)\end{array}$ & $59.99 \pm 38.2(52)$ & $66.61 \pm 30.3(240)$ \\
\hline
\end{tabular}

Data are $n$, mean \pm SD, or mean \pm SD $(N)$ values

6-MP 6-mercaptopurine, NA not available

$p$ values are for $t$-tests or $^{2}$ tests as appropriate. ${ }^{*} p<0.05$

a Data for age were not available for one subject

clinical endpoint. The recorded 6-MP dose per square meter of the body surface over a 12-week cycle was used to define the actual administered dose as a percentage of the planned dose as the last-cycle DIP. Since East Asian ancestry requires significantly lower 6-MP dose intensity compared to the other ethnic groups [5], patients who require less than $25 \%$ of the protocol planned dose were classified as MP-intolerant groups [6]. We have previously presented a detailed description of the subjects and a summary of the measurements [7]. The present study was approved by the SNUH, AMC, and SMC institutional review boards. Written informed consent was obtained from each participant.

\section{Whole-exome sequencing and primary data analysis}

WES data obtained from the 320 pediatric ALL patients were analyzed in a bioinformatics pipeline as we have described previously [7]. Two missense NUDT15 variants with no officially designated star alleles were confirmed using Sanger sequencing, and false positive variant calls were removed in the further analysis. According to the CPIC guideline updated in February 2019, where the activity of NUDT15*9 for 6-MP was changed from 'uncertain' to 'no function' [1], one patient 
was reclassified as a poor metabolizer of NUDT15. The present study analyzed the 240 normal metabolizers (188 and 52 in the discovery and replication cohorts, respectively) of both NUDT15 and TPMT according to their star-allele genotypes. In the discovery phase, functional consequences of variants were predicted using SnpEFF (http://snpeff.sourceforge.net) [8], and only variants predicted to have a strong effect on gene function (missense, nonsense, splice-site, frameshift, and in-frame insertion and deletion variants) were chosen (Fig. 1).

Age- and sex-adjusted multivariate linear regression analyses of the DIP model identified 185 variants $(p<0.01)$ in 159 genes, of which 12 candidate variants (in 12 genes, Table 2) determined by 2 in silico prediction methods [i.e., SIFT (sorting intolerant from tolerant) [9] score $\leq 0.05$ and CADD (combined annotation-dependent depletion) [10] score $\geq 25$ ] were evaluated in the external replication cohort using multiple regression analyses (Fig. 1). We identified 1 final candidate variant, rs3821169, in the gene encoding Cysteine-Rich Transmembrane BMP Regulator 1 (CRIM1) that exhibited statistically significant associations for the last-cycle DIP $(p<0.05)$ in both additive and recessive models. Finally, we performed genotyping assays to experimentally validate the identified candidate variant.

\section{Subsequent genotyping and validation}

To confirm the genotype calls of the final candidate variant, rs3821169, we performed SNPtype (Fluidigm, San Francisco, CA) assays for 118 subjects with 2 control samples whose blood DNA was available after the WES. In the SNPtype assay, genomic DNA flanking the SNP of interest was amplified by PCR with an STA primer set and Qiagen $2 \times$ Multiplex PCR Master Mix (Qiagen) in a total reaction volume of $5 \mu \mathrm{L}$ that contained $40 \mathrm{ng}$ of genomic DNA. PCR was carried out as follows: 1 cycle at $95^{\circ} \mathrm{C}$ for $15 \mathrm{~min}$, and then 14 cycles at $95^{\circ} \mathrm{C}$ for $15 \mathrm{~s}$ and $60{ }^{\circ} \mathrm{C}$ for $4 \mathrm{~min}$. After amplification, STA products were diluted 1:100 in DNA suspension buffer, and $2.5 \mu \mathrm{L}$ of the diluted STA products was added to a sample premix that contained $3 \mu \mathrm{L}$ of $2 \times$ Fast Probe Master Mix, $0.3 \mu \mathrm{L}$ of SNPtype $20 \times$ Sample Loading Reagent, $0.1 \mu \mathrm{L}$ of SNPtype Reagent, and $0.036 \mu \mathrm{L}$ of ROX. After the assay premix and sample premix were loaded into the 192.24 Dynamic Array, the SNPtype assay reaction was carried out as follows: 1 cycle at $95^{\circ} \mathrm{C}$ for $5 \mathrm{~min} ; 1$ cycle at $95^{\circ} \mathrm{C}$ for $15 \mathrm{~s}, 64{ }^{\circ} \mathrm{C}$ for $45 \mathrm{~s}$, and $72{ }^{\circ} \mathrm{C}$ for $15 \mathrm{~s} ; 1$ cycle at $95^{\circ} \mathrm{C}$ for $15 \mathrm{~s}, 63^{\circ} \mathrm{C}$ for $45 \mathrm{~s}$, and $72{ }^{\circ} \mathrm{C}$ for $15 \mathrm{~s} ; 1$ cycle at $95^{\circ} \mathrm{C}$ for $15 \mathrm{~s}, 62^{\circ} \mathrm{C}$ for $45 \mathrm{~s}$, and $72{ }^{\circ} \mathrm{C}$ for $15 \mathrm{~s} ; 1$ cycle at $95^{\circ} \mathrm{C}$ for $15 \mathrm{~s}, 61{ }^{\circ} \mathrm{C}$ for $45 \mathrm{~s}$, and $72{ }^{\circ} \mathrm{C}$ for $15 \mathrm{~s} ; 34$ cycles at $95{ }^{\circ} \mathrm{C}$ for $15 \mathrm{~s}, 60{ }^{\circ} \mathrm{C}$ for $45 \mathrm{~s}$ and $72{ }^{\circ} \mathrm{C}$ for $15 \mathrm{~s}$; and 1

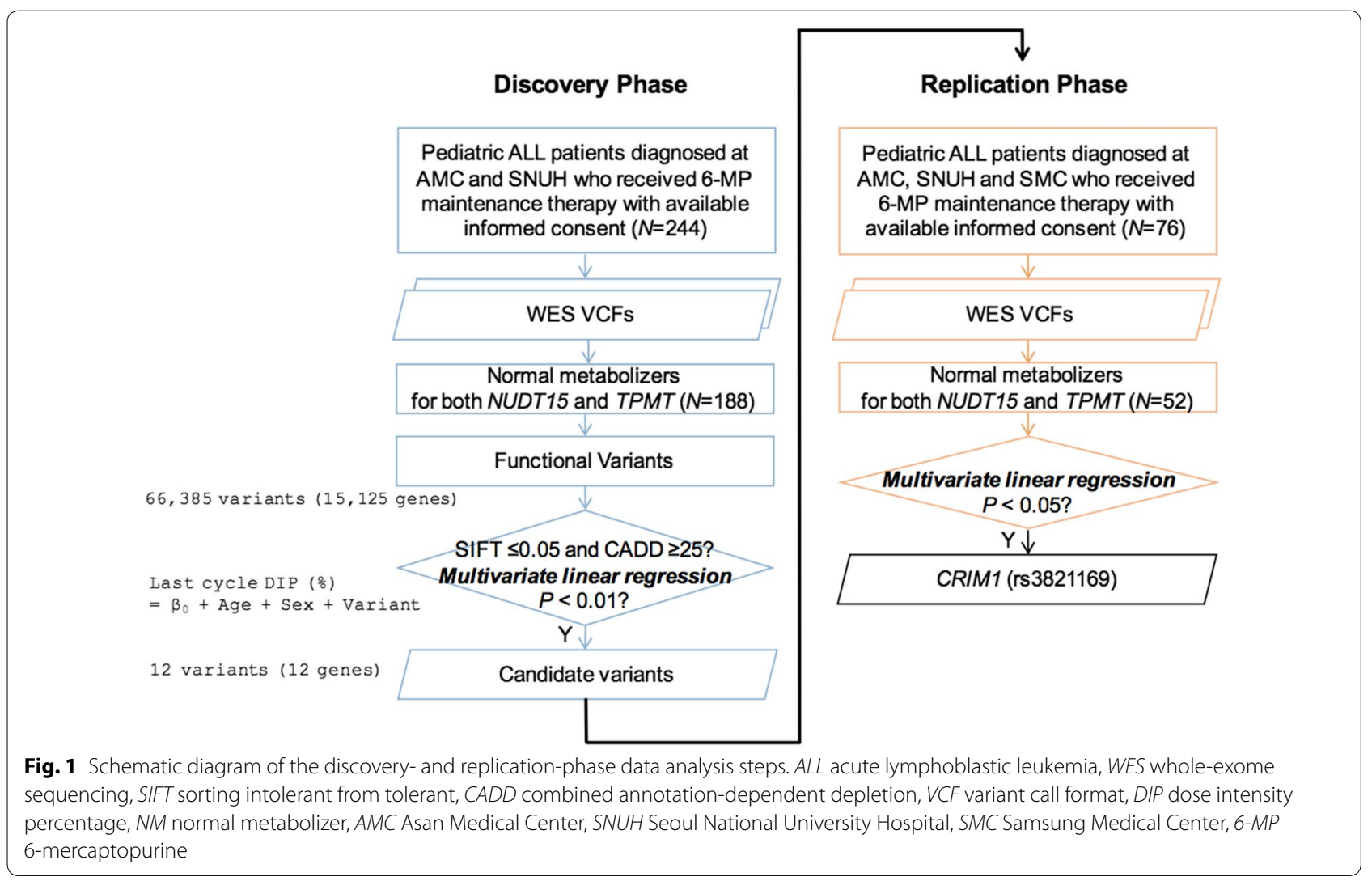




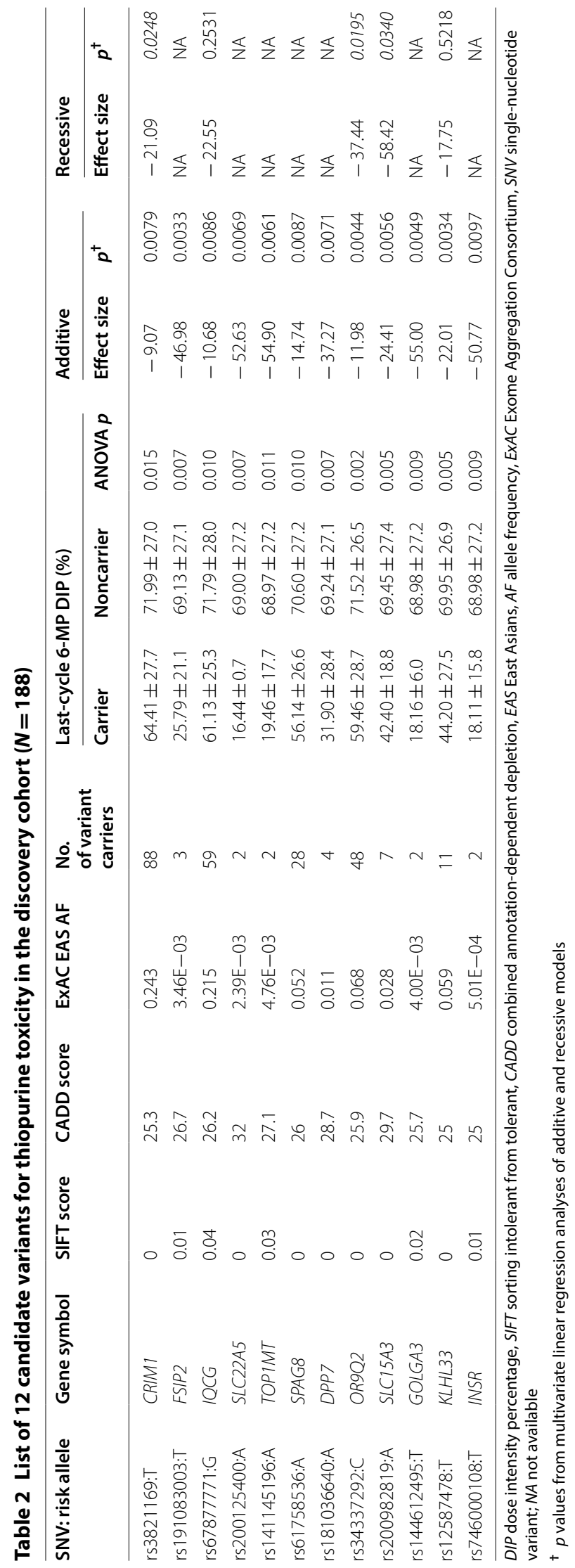


cycle at $25{ }^{\circ} \mathrm{C}$ for $10 \mathrm{~s}$. The genotyping test was carried out using Fluidigm SNP Genotyping Analysis software (version 4.0.1, Fluidigm).

For the two missense variants in NUDT15 with no officially designated star alleles, we performed independent validation using Sanger sequencing. Exon 1 of NUDT15, including the rs780144127 and 13: $48611982 \mathrm{~A}>\mathrm{G}$, was amplified for Sanger sequencing. PCR assays were performed directly to amplify 20 ng of the genomic DNA samples to collect the target regions using the oligoprimer pairs. Reaction parameters were as follows: $95^{\circ} \mathrm{C}$ for $5 \mathrm{~min}$, followed by 35 cycles of $95^{\circ} \mathrm{C}$ for $30 \mathrm{~s}, 58^{\circ} \mathrm{C}$ for $30 \mathrm{~s}, 72{ }^{\circ} \mathrm{C}$ for $1 \mathrm{~min}$ and $72{ }^{\circ} \mathrm{C}$ for $10 \mathrm{~min}$. RBC HiYield Gel/PCR DNA Mini Kit was used to purify the DNA in the PCR products (Taipai county 220, Taiwan). After purification, the PCR samples were directly sequenced using an ABI 3100 semi-automated sequencing analyzer (Applied Biosystems, Lincoln Center Drive Foster City, CA, USA). The DNA sequences were analyzed using FinchTV version 1.4.0 (Geospiza, Inc., Seattle, WA, USA).

\section{Single- and multigene prediction accuracies for thiopurine toxicity}

Gene-wise variant burden (GVB) analysis was performed to evaluate the aggregated impact of both common and rare variants $[7,11,12]$. The GVB of a coding gene for each individual was defined as the geometric mean of the SIFT scores of the coding variants (SIFT score $<0.7$ ) in the coding gene, where $\mathrm{GVB}^{G}$ denotes the GVB score of gene $G$. The powers of $\mathrm{GVB}^{N U D T 15}, \mathrm{GVB}^{T P M T}$, and $\mathrm{GVB}^{\text {CRIM1 }}$ for predicting the last-cycle 6-MP DIP were systematically evaluated by analyzing ROC (receiver operating characteristic) curves across seven DIP cutoffs (i.e., $15 \%, 25 \%, 35 \%, 45 \%, 60 \%, 80 \%$, and $100 \%$ ) in terms of the areas under the ROC curves (AUCs) in the discovery, replication, and combined cohorts before and after controlling for the effects of the other two genes. Multigene effects were systematically evaluated by defining $\mathrm{GVB}^{A, B}$ as the geometric mean of $\mathrm{GVB}^{A}$ and $\mathrm{GVB}^{B}$.

All statistical analyses were performed using the R statistical package (version 3.5.1). To correctly evaluate the recessive model for the CRIM1 variant in this study, the effect of the heterozygous rs3821169 variant was ignored when computing $\mathrm{GVB}^{\text {CRIMI }}$.

\section{Star-allele diplotype vs. gene-wise variant burden}

The traditional pharmacogenetic star-allele assignment system classifies study subjects into categorical molecular-phenotype groups. However, novel pharmacogenes do not yet have star-allele assignments. Genes do not work alone, and the categorical nature of traditional starallele-based molecular phenotyping makes it nontrivial to consistently evaluate the multigene pharmacogenetic effects of a drug. The GVB method assigns a corresponding quantitative score for each gene to each individual, enabling the consistent quantization of multigene GVB scores of an individual into a personalized drug GVB score. To evaluate the clinical utility of the GVB method, we systematically compared the traditional star-allelebased molecular phenotyping method with single- and multigene GVB methods for predicting 6-MP intolerance in pediatric ALL patients (Tables 3 and 4).

\section{Results}

Description of patients

It was determined that 240 of the 320 pediatric ALL patients (188 in the 244 discovery cohort and 52 in the 76 replication cohort) did not carry CPIC-reported pathogenic (or pharmacogenetic) variants in either NUDT15

Table 3 Prediction accuracies of CRIM1 rs3821169 variant for thiopurine toxicity measured by the tolerated last-cycle 6-MP DIP in pediatric ALL subjects with both wild-type NUDT15 and TPMT

\begin{tabular}{|c|c|c|c|c|c|c|c|c|c|}
\hline \multirow[t]{2}{*}{ Phase } & \multirow{2}{*}{$\begin{array}{l}\text { rs3821169 } \\
\text { homozygote } \\
\text { carriers }\end{array}$} & \multicolumn{3}{|l|}{ DIP } & \multirow[t]{2}{*}{ Sensitivity } & \multirow[t]{2}{*}{ Specificity } & \multirow[t]{2}{*}{ PPV } & \multirow[t]{2}{*}{ NPV } & \multirow[t]{2}{*}{ Accuracy } \\
\hline & & $\leq 25 \%$ & $>25 \%$ & Total & & & & & \\
\hline \multirow[t]{3}{*}{ Discovery } & $(+)$ & 2 & 7 & 9 & 0.222 & 0.961 & 0.222 & 0.961 & 0.926 \\
\hline & $(-)$ & 7 & 172 & 179 & & & & & \\
\hline & Total & 9 & 179 & 188 & & & & & \\
\hline \multirow[t]{3}{*}{ Replication } & $(+)$ & 3 & 0 & 3 & 0.250 & 1.000 & 1.000 & 0.816 & 0.827 \\
\hline & $(-)$ & 9 & 40 & 49 & & & & & \\
\hline & Total & 12 & 40 & 52 & & & & & \\
\hline \multirow[t]{3}{*}{ Combined } & $(+)$ & 5 & 7 & 12 & 0.238 & 0.968 & 0.417 & 0.930 & 0.904 \\
\hline & $(-)$ & 16 & 212 & 228 & & & & & \\
\hline & Total & 21 & 219 & 240 & & & & & \\
\hline
\end{tabular}


Table 4 Comparison of star-allele-based diplotyping vs. the gene-wise variant burden (GVB) method for predicting thiopurine toxicity in pediatric ALL subjects

\begin{tabular}{|c|c|c|c|c|c|c|c|c|c|c|}
\hline \multirow[t]{2}{*}{ Phase } & \multirow[t]{2}{*}{ Method } & \multirow{2}{*}{$\begin{array}{l}\text { Molecular } \\
\text { phenotype }\end{array}$} & \multicolumn{3}{|c|}{ Last-cycle 6-MP DIP } & \multirow[t]{2}{*}{ Sensitivity } & \multirow[t]{2}{*}{ Specificity } & \multirow[t]{2}{*}{ PPV } & \multirow[t]{2}{*}{ NPV } & \multirow[t]{2}{*}{ Accuracy } \\
\hline & & & $\leq 25 \%$ & $>25 \%$ & Total & & & & & \\
\hline \multirow[t]{7}{*}{ Discovery } & \multirow[t]{2}{*}{ CPIC NUDT15 and TPMT metabolizer } & $P M+\mid M$ & 10 & 46 & 56 & \multirow[t]{2}{*}{0.526} & \multirow[t]{2}{*}{0.796} & \multirow[t]{2}{*}{0.179} & \multirow[t]{2}{*}{0.952} & \multirow[t]{2}{*}{0.775} \\
\hline & & NM & 9 & 179 & 188 & & & & & \\
\hline & \multirow[t]{2}{*}{$\mathrm{GVB}^{N U D T 15, T P M T}$} & $\leq 0.3$ & 10 & 42 & 52 & \multirow[t]{2}{*}{0.526} & \multirow[t]{2}{*}{0.813} & \multirow[t]{2}{*}{0.192} & \multirow[t]{2}{*}{0.953} & \multirow[t]{2}{*}{0.791} \\
\hline & & $>0.3$ & 9 & 183 & 192 & & & & & \\
\hline & \multirow[t]{3}{*}{$\mathrm{GVB}^{\text {NUDT15,TPMT,CRIM1 }}$} & $\leq 0.3$ & 11 & 32 & 43 & \multirow[t]{3}{*}{0.579} & \multirow[t]{3}{*}{0.858} & \multirow[t]{3}{*}{0.256} & \multirow[t]{3}{*}{0.960} & \multirow[t]{3}{*}{0.836} \\
\hline & & $>0.3$ & 8 & 193 & 201 & & & & & \\
\hline & & Total & 19 & 225 & 244 & & & & & \\
\hline \multirow[t]{7}{*}{ Replication } & \multirow[t]{2}{*}{ CPIC NUDT15 and TPMT metabolizer } & $P M+\mid M$ & 13 & 11 & 24 & \multirow[t]{2}{*}{0.520} & \multirow[t]{2}{*}{0.784} & 0.542 & 0.769 & 0.697 \\
\hline & & NM & 12 & 40 & 52 & & & & & \\
\hline & $\mathrm{GVB}^{\text {NUDT15,TPMT }}$ & $\leq 0.3$ & 13 & 10 & 23 & 0.520 & 0.804 & 0.565 & 0.774 & 0.711 \\
\hline & & $>0.3$ & 12 & 41 & 53 & & & & & \\
\hline & $\mathrm{GVB}^{\text {NUDT15,TPMT,CRIM1 }}$ & $\leq 0.45$ & 16 & 10 & 26 & 0.640 & 0.804 & 0.615 & 0.820 & 0.750 \\
\hline & & $>0.45$ & 9 & 41 & 50 & & & & & \\
\hline & & Total & 25 & 51 & 76 & & & & & \\
\hline Combined & CPIC NUDT15 and TPMT metabolizer & $P M+\mid M$ & 23 & 57 & 80 & 0.523 & 0.794 & 0.288 & 0.913 & 0.756 \\
\hline & & NM & 21 & 219 & 240 & & & & & \\
\hline & $\mathrm{GVB}^{\text {NUDT15,TPMT }}$ & $\leq 0.3$ & 23 & 52 & 75 & 0.523 & 0.811 & 0.307 & 0.914 & 0.772 \\
\hline & & $>0.3$ & 21 & 224 & 245 & & & & & \\
\hline & $\mathrm{GVB}^{\text {NUDT15,TPMT,CRIM1 }}$ & $\leq 0.45$ & 28 & 60 & 88 & 0.636 & 0.783 & 0.318 & 0.931 & 0.763 \\
\hline & & $>0.45$ & 16 & 216 & 232 & & & & & \\
\hline & & Total & 44 & 276 & 320 & & & & & \\
\hline
\end{tabular}

Prediction accuracies for the last-cycle 6-MP DIP of star-allele-based Clinical Pharmacogenetics Implementation Consortium (CPIC) practice guidelines on NUDT15 and TPMT were compared with the quantitative GVB ${ }^{\text {NUDT15,TPMT }}$ and GVB ${ }^{\text {NUDT15,TPMT,CRIM1 }}$ methods in the discovery, replication, and combined cohorts. GVB cutoffs were determined by maximizing Youden's index

IM intermediate metabolizer, PM poor metabolizer

or TPMT. Table 1 presents the clinical characteristics of the 240 subjects who carried both WT NUDT15 and TPMT. Compared to the non-both-WT subjects $(N=80)$, the both-WT subjects $(N=240)$ demonstrated significantly higher tolerated last-cycle DIPs in the discovery cohort $[68.44 \pm 27.6$ vs. $54.14 \pm 29.9($ mean $\pm S D)$, $p=0.002$ by $\mathrm{t}$-test], the replication cohort $(59.99 \pm 38.2$ vs. $33.36 \pm 28.7, p=0.001$ by $t$-test), and the two cohorts combined. These findings confirm the well-established effects of NUDT15 and TPMT pharmacogenetic variants on thiopurine toxicity in pediatric ALL.

However, Table 1 also demonstrates that 4.8\% (9 of 188 ) and $23.1 \%$ (12 of 52) of the both-WT subjects in the discovery and replication cohorts, respectively, were classified as a high-risk group for thiopurine toxicity (DIP $<25 \%$ ), while $63.8 \%$ (120 of 188 ) and $46.2 \%$ (24 of 52), respectively, of the both-WT subjects were classified as a moderate-risk group (DIP $<80 \%$ ). The difference in the frequency of high-risk subjects between the discovery and replication cohorts is probably due to the lack of available replication data. Overall, $68.8 \%(N=165)$ of the 240 subjects who carried both WT NUDT15 and
TPMT still demonstrated as-yet-unexplained thiopurine response variability.

\section{Candidate genes for thiopurine toxicity beyond NUDT15 and TPMT}

Age- and sex-adjusted variant-level multivariate linear regression analyses were performed for the 66,385 variants predicted to have strong effects on gene function (i.e., 64,238 missense, 1249 nonsense, 552 splice-site, 332 frameshift, and 4 in-frame insertion and deletion variants) for the both-WT subjects $(N=188)$ in the discovery cohort $(N=244)$ (Fig. 1). Twelve candidate variants in 12 genes were selected by applying a significance cutoff of $p<0.01$ and 2 in silico prediction methods for variant function (SIFT score $\leq 0.05$ and CADD score $\geq 25$ ). Due to the small number of study samples and the rarity of the deleterious variants for full correction of multiple hypotheses, a less-stringent $p$ cutoff was applied for the discovery-phase candidate variant analysis.

Table 2 lists the 12 candidate variants for thiopurine toxicity. Only the rs3821169 variant in CRIM1 was successfully replicated for statistically significant 
associations with lower last-cycle 6-MP DIP by multivariate regression analyses in both additive $(p=0.0483)$ and recessive $(p=0.0132)$ models (Additional file 1 : Table S1). Note that a recessive model could not be correctly applied to 10 of the 12 candidate variants due to the small number of replication subjects along with low allele frequencies (Additional file 1: Table S1).

\section{Evaluation of the association between the CRIM1 variant and thiopurine toxicity}

Carriers of the CRIM1 rs3821169 variant demonstrated significantly lower last-cycle 6-MP DIPs in the discovery cohort $(p=0.007)$, replication cohort $(p=0.048)$, and combined cohort $(p<0.001)$ by multivariate linear regression under an additive model (Fig. 2). Strong associations of this variant under a recessive model were also found for the discovery, replication, and combined cohorts $(p=0.025,0.013$, and 0.001 , respectively). The statistical power to detect associations in the replication cohort was lost under a dominant model $(p=0.028,0.224$, and 0.013 ), which was at least partly due to the small number of subjects in that cohort. Given the high frequency of CRIM1 rs3821169 carriers (46.8\%) in East Asian subjects, we focused on the homozygote (or recessive) effect of this variant on thiopurine toxicity in the present study.
To evaluate the consistency of the statistical association between the rs 3821169 variant and thiopurine toxicity, the candidate variant association was tested across all threshold cutoffs of thiopurine toxicity (i.e., Group $1\left(G_{1}\right) \leq 70 \%$, $G_{2} \leq 60 \%, G_{3} \leq 45 \%, G_{4} \leq 35 \%, G_{5} \leq 25 \%$, and $G_{6}<15 \%$ DIPs) by defining two control groups: (1) $G_{0}$, comprising the 89,21 , and 110 ALL patients with DIP $>70 \%$ in the discovery, replication, and combined cohorts, respectively, and (2) external healthy controls, obtained from the 504 East Asians in the 1000 Genomes Project [13] (Additional file 1: Table S2). Fisher's exact test for dominant and recessive models and the Cochran-Armitage trend test (CATT) were applied. Four of the six comparison groups for the last-cycle 6-MP DIP in both Fisher's exact tests (recessive model) and CATTs demonstrated consistent statistical significances for both the $G_{0}$ and East-Asian control groups (Additional file 1: Table S2). We experimentally validated and confirmed the rs3821169 genotypes using the Fluidigm genotyping method in 118 subjects for whom blood samples were available, which revealed $97.4 \%$ concordance.

\section{Multigene effects of NUDT15, TPMT, and CRIM1 on thiopurine toxicity}

To evaluate the additive effects of the novel CRIM1 rs3821169 variant relative to the well-known NUDT15 and

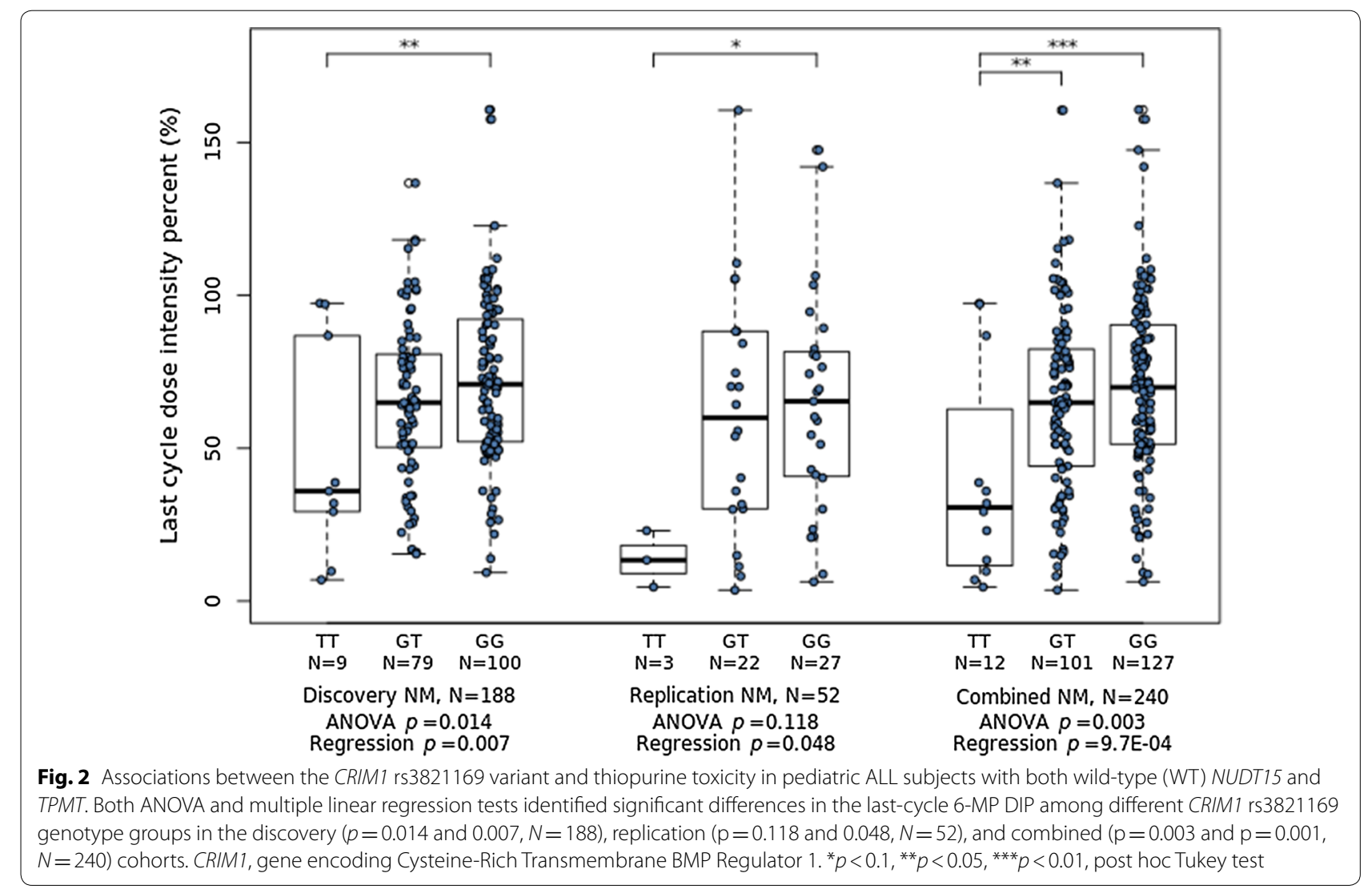


TPMT pharmacogenetic effects, GVB-based ROC analyses were performed before and after introducing the homozygous CRIM1 rs3821169 variant for the entire cohort of 320 pediatric ALL patients (Fig. 3 and Additional file 1: Figures S1-S3). Figure 3 shows the AUCs representing the diagnostic accuracies of the traditional two-gene prediction model (GVB ${ }^{\text {NUDT15,TPMT }}$, left panels in the figure) and the newly introduced three-gene prediction model (GVB ${ }^{\text {NUDTI5,TPMT,CRIM1, right panels in the figure) across }}$ all seven DIP cutoffs ( $\leq 15 \%, \leq 25 \%, \leq 35 \%, \leq 45 \%, \leq 60 \%$, $\leq 80 \%$, and $\leq 100 \%)$ in the discovery $(N=244)$, replication $(N=76)$, and combined $(N=320)$ pediatric ALL cohorts. $\mathrm{GVB}^{\text {NUDT15,TPMT,CRIM1 }}$ outperformed the traditional twogene model GVB ${ }^{\text {NUDT15,TPMT }}$ at all threshold cutoffs in the discovery, replication, and combined cohorts (e.g., AUC ${ }^{<15 \%}=0.810$ vs. $0.706,0.697$ vs. 0.600 , and 0.754 vs. 0.658 , respectively; $\mathrm{AUC}^{<25 \%}=0.739$ vs. $0.684,0.728$ vs. 0.633 , and 0.737 vs. 0.667 , respectively), with the only exception being $\mathrm{DIP}<100 \%$ in the replication cohort $\left(\mathrm{AUC}^{<100 \%}=0.642\right.$ vs. 0.676) (Fig. 3).

More importantly, dose-response relationships for predicting 6-MP intolerance were observed. A lower DIP was associated with a higher AUC for both GVB ${ }^{\text {NUDTI5,TPMT }}$ and $\mathrm{GVB}^{\text {NUDT15,TPMT,CRIM1 }}$ (Fig. 3). For example, for the discovery phase of $\mathrm{GVB}^{\text {NUDT15,TPMT,CRIM1 }}, \mathrm{AUC}^{<15 \%}=0.810$ was higher than $\mathrm{AUC}^{<25 \%}=0.739$, which was higher than AUC ${ }^{<35 \%}=0.624$ (Fig. 3). Given the high frequency of rs 3821169 carriers (46.8\%) in East Asian subjects, we focused on the homozygote (or recessive) effect of the CRIM1 rs3821169

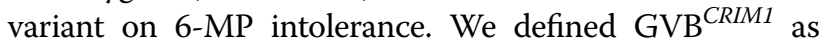
the GVB score of CRIM1 while ignoring heterozygous rs3821169 and considering only homozygous rs3821169.

\section{Contributions of single genes to thiopurine toxicity}

Figure 4 demonstrates the diagnostic prediction accuracies for thiopurine toxicity for each of CRIM1, NUDT15, and TPMT after controlling for the effects of the other two genes in the entire cohort $(N=320)$. The AUCs of $\mathrm{GVB}^{C R I M 1}$ were measured for the 240 subjects who carried both WT NUDT15 and TPMT (left panels in Fig. 4), while the AUCs of GVB ${ }^{N U D T 15}$ were measured for the 294 subjects with WT TPMT and nonhomozygote carriers of the CRIM1 rs3821169 variant (middle panels in Fig. 4). The AUCs of GVB ${ }^{T P M T}$ were measured for the 236 subjects with WT NUDT15 and nonhomozygote carriers of the CRIM1 rs3821169 variant (right panels in Fig. 4). The prediction accuracies were measured for each of the discovery, replication, and combined cohorts (upper, middle, and lower panels in Fig. 4, respectively).

Overall, NUDT15 exhibited the best single-gene prediction accuracies for the last-cycle 6-MP DIP for the DIP $<25 \%$ cutoff in the discovery $(\mathrm{AUC}=0.656$, $N=224$ ), replication (AUC $=0.697, N=70$ ), and combined (AUC $=0.690, N=294$ ) cohorts. The recessive CRIM1 model exhibited performances in the discovery $(\mathrm{AUC}=0.623, N=188)$, replication $(\mathrm{AUC}=0.696$, $N=52)$ and combined (AUC $=0.658, N=240$ ) cohorts that were comparable to NUDT15, which is the bestestablished and strongest predictor of 6-MP intolerance for East Asians. TPMT exhibited poor performance in the present study, which can be explained by the very low frequencies of TPMT variants in East Asian compared to European populations.

More importantly, each of NUDT15 and CRIM1 exhibited a dose-response relationship for predicting thiopurine toxicity. A lower DIP was associated with a higher AUC for both NUDT15 and CRIM1 (Fig. 4). Overall, it is suggested that the novel CRIM1 rs3821169 variant (in its homozygote form) exerts both independent and additive pharmacogenetic effects (to the known NUDT15 and TPMT genes) to thiopurine toxicity, especially in East Asian populations with a high allele frequency (0.243 in the Exome Aggregation Consortium database; Table 2). Additional file 1: Figures S4-S6 provide the results of further detailed analyses of single gene effects on 6-MP intolerance, exhibiting consistent results, as depicted in Fig. 4.

\section{Evaluation of the prediction accuracies of NUDT15, TPMT, and CRIM 1}

Table 3 presents the diagnostic accuracies of the CRIM1 rs3821169 homozygote variant for the last-cycle 6-MP DIP in the discovery (0.926), replication (0.827), and combined (0.904) cohorts. The CRIM1 rs3821169 homozygosity itself exhibited relatively low sensitivities $(0.222-0.250)$ and positive predictive values $(0.222-$ $1.000)$, and relatively high specificities $(0.961-1.000)$ and negative predictive values $(0.816-0.961)$.

The current CPIC pharmacogenetic testing guideline for 6-MP in treating pediatric ALL patients applies star-allele-based diplotypes of TPMT and NUDT15 $[3,4]$. A star allele is defined and/or inferred by a set

\footnotetext{
(See figure on next page.)

Fig. 3 Improvement prediction accuracies for thiopurine toxicity by introducing CRIM1 into the well-established NUDT15 and TPMT in 320 pediatric ALL subjects. Prediction accuracies (measured in AUCs) for the last-cycle 6-MP DIP of the three-gene model (NUDT15, TPMT and CRIM1) (right panels) outperformed the traditional two-gene model (NUDT15 and TPMT) (left panels) across all seven DIP cutoffs ( $\leq 15 \%, \leq 25 \%, \leq 35 \%, \leq 45 \%$, $\leq 60 \%, \leq 80 \%$, and $\leq 100 \%)$ in the discovery $(N=244)$, replication $(N=76)$, and combined $(N=320)$ pediatric ALL cohorts. $95 \%$ confidence intervals are in square brackets. GVB, gene-wise variant burden; $A \cup C$, area under the receiver operating characteristic curve
} 

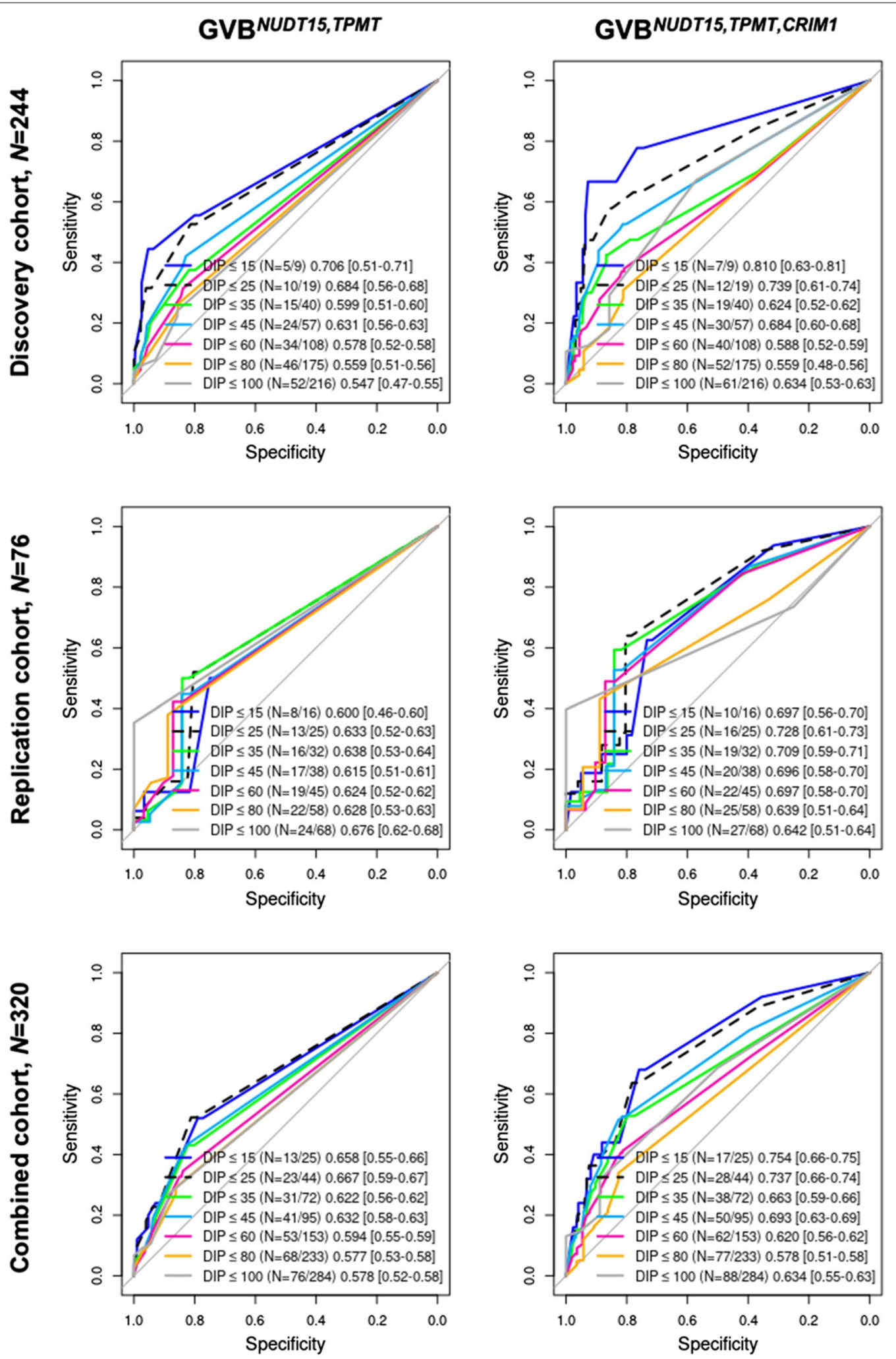


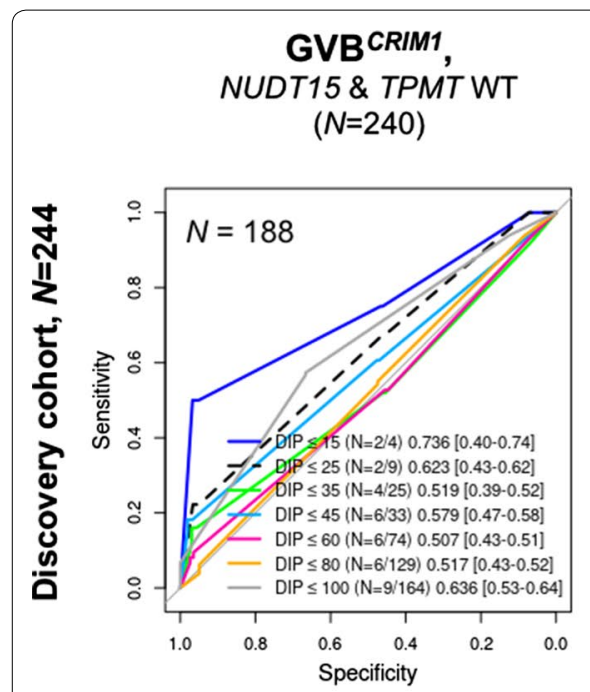

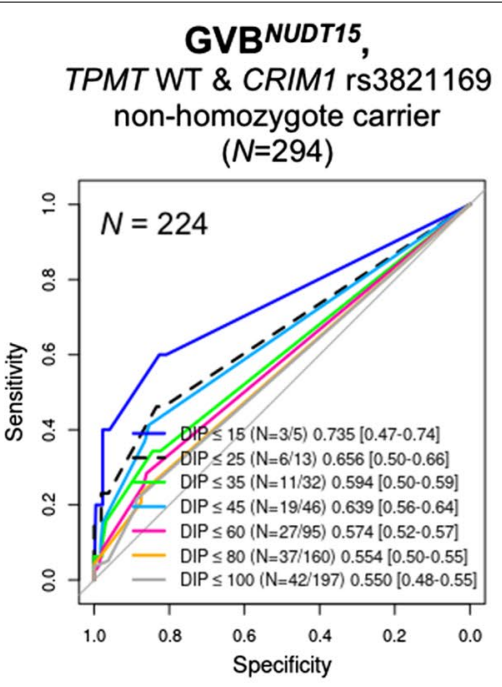

\section{GVBTPMT \\ NUDT15 WT \& CRIM1 rs3821169 non-homozygote carrier}

$(N=236)$
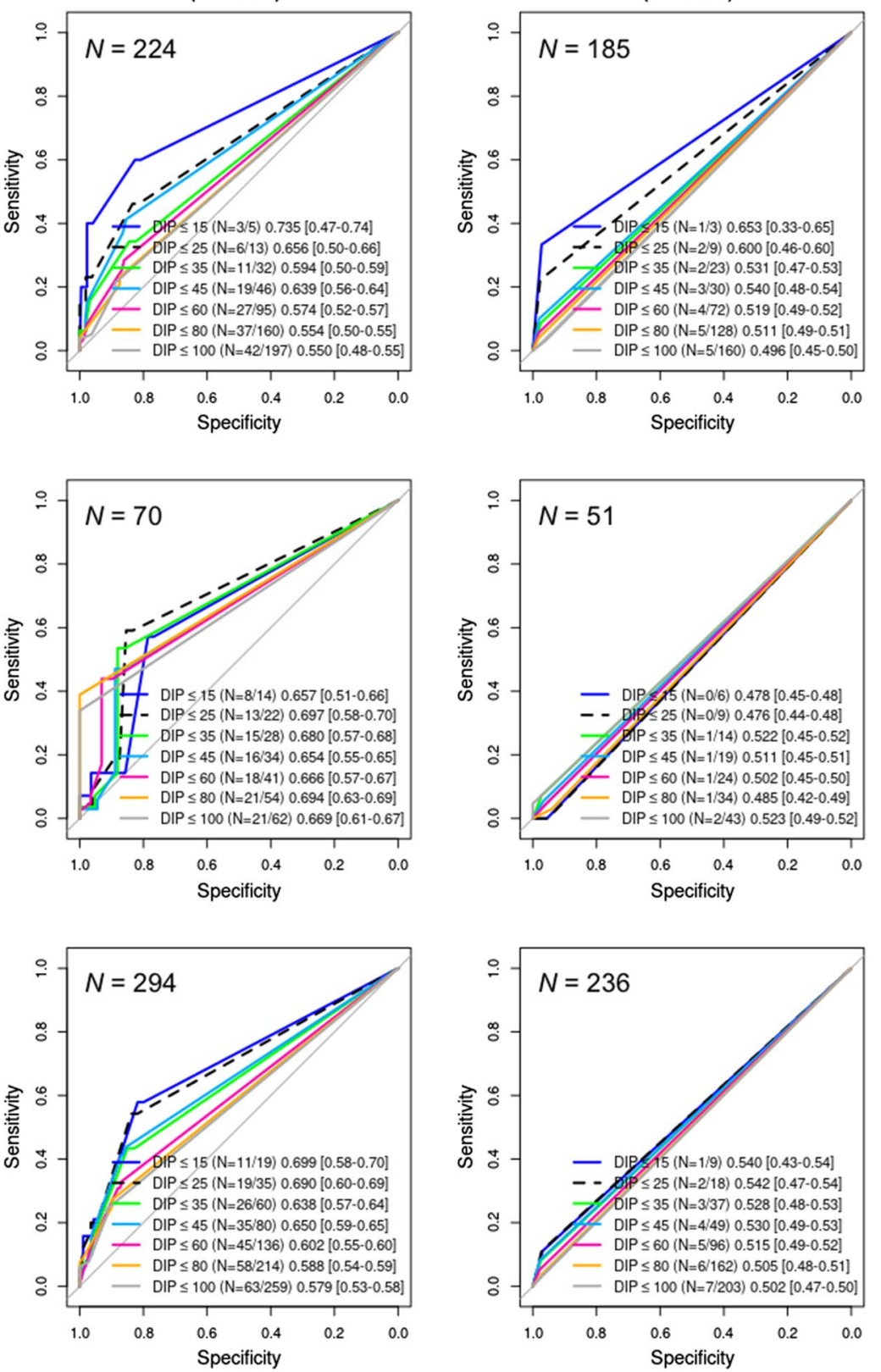

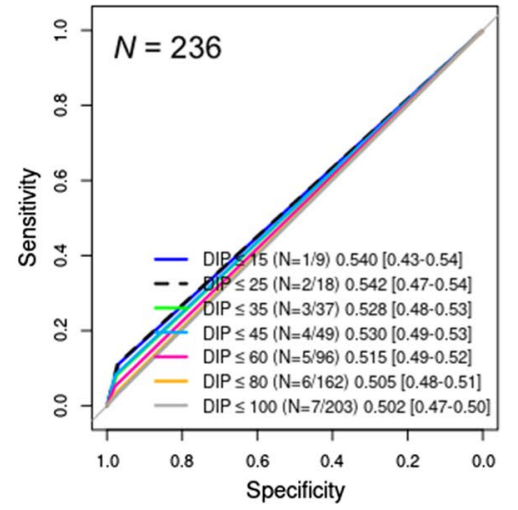

Fig. 4 Evaluation of the single-gene contribution of CRIM1, NUDT15, and TPMT in predicting thiopurine toxicity after controlling for the effects of the other two genes in pediatric ALL subjects. Prediction accuracies of GVB ${ }^{\text {CRIM1 }}$, GVB ${ }^{\text {NUDT15}}$, and GVB ${ }^{\text {TPMT }}$ for predicting seven cutoffs of the last-cycle 6 -MP DIPs ( $\leq 15 \%, \leq 25 \%, \leq 35 \%, \leq 45 \%, \leq 60 \%, \leq 80 \%$, and $\leq 100 \%$ ) were measured using AUCs after controlling for the effects of the other two genes. $95 \%$ confidence intervals are in square brackets

of genotypes. CPIC guidelines generally do not provide a specific instruction on how to combine multigene interactions for the categorical star-allele classes. Moreover, there are no star-allele assignments for
CRIM1 yet, so evaluating the clinical utility of applying multigene pharmacogenetic testing remains a nontrivial problem. To evaluate the utility of the GVB scoring method for combining multigene effects, we 
systematically compared the diagnostic accuracies of the traditional star alleles of NUDT15 and TPMT with GVB-quantitation-based GVB ${ }^{N U D T 15, T P M T}$ as well as GVB ${ }^{\text {NUDT15,TPMT,CRIM1 }}$ (Table 4). The optimal cutoff for the GVB score was determined by maximizing Youden's index (Additional file 1: Figure S7).

Table 4 demonstrates that GVB ${ }^{\text {NUDT15,TPMT }}$ yielded slightly better prediction accuracies than the traditional star-allele-based diplotyping method in the discovery $(0.791$ vs. 0.775$)$, replication $(0.711$ vs. 0.697$)$, and combined $(0.772$ vs. 0.756$)$ cohorts, along with improvements in sensitivity, specificity, and positive and negative predictive values. Given that a designated star allele for CRIM1 is not available yet, we created a three-gene prediction model: GVB ${ }^{N U D T 15, T P M T, C R I M 1}$ outperformed the traditional star-allele-based NUDT15 and TPMT diplotyping method in the discovery (0.836 vs. 0.775$)$, replication $(0.750$ vs. 0.697$)$, and combined (0.763 vs. 0.756) cohorts, along with exhibiting improvements in sensitivity, specificity, and positive and negative predictive values (Table 4). At the clinical endpoint of the last-cycle 6 -MP DIP $<25 \%$, $\mathrm{GVB}^{\text {NUDT15,TPMT,CRIM1 }}$ also outperformed the traditional star-allele method in terms of AUC (0.737 vs. 0.665 , Fig. 3 ), prediction accuracy (0.763 vs. 0.756 ), sensitivity ( 0.636 vs. 0.523$)$, positive predictive value ( 0.318 vs. 0.288$)$, and negative predictive value $(0.931$ vs. 0.913) (Table 4).

$\mathrm{GVB}^{\text {NUDT15,TPMT,CRIM1 } 1 \text { also outperformed GVB }}{ }^{\text {NUDT15,TPMT }}$ in the discovery, replication, and combined cohorts in terms of sensitivity $(0.579$ vs. $0.526,0.640$ vs. 0.520 , and 0.636 vs. 0.523 , respectively), positive predictive value ( 0.256 vs. 0.192 , 0.615 vs. 0.563 , and 0.318 vs. 0.307 ), and negative predictive value $(0.960$ vs. $0.953,0.820$ vs. $0.774,0.931$ vs. 0.914$)$. Specificity ( 0.858 vs. $0.813,0.804$ vs. $0.804,0.783$ vs. 0.811$)$ and accuracy ( 0.836 vs. $0.791,0.750$ vs. 0.711 , and 0.763 vs. 0.772$)$ were improved in the discovery and replication cohorts, but slightly worse in the combined cohort (Table 4). The distribution of nonsynonymous variants in NUDT15, TPMT, and CRIM1 genes for 320 ALL patients is summarized in Additional file 1: Table S3.

\section{Discussion}

CRIM1 is a cell-surface transmembrane protein that resembles developmentally important proteins which are known to interact with bone morphogenetic proteins (BMPs). A role of CRIM1 in drug resistance has been suggested by previous studies $[14,15]$ revealing that the level of mRNA expression of CRIM1 is high in resistant leukemic cells. This affects the levels of BMPs, suggesting that CRIM1 regulates the growth and differentiation of hematopoietic cells. The Genomics of Drug Sensitivity in Cancer study [16] found that rs3821169 heterozygous cases showed lower mRNA expression levels compared to the WT cases (Additional file 1: Figure S8, $p=0.095$ by one-tailed $t$-test). It is suggested that subjects carrying this variant display drug-sensitive responsiveness, although the potential for loss of function of the corresponding protein was not predictable since no homozygous variant was found in the data set, probably due to the low allele frequency of rs3821169 in Western populations. Further experimental validation is needed to determine how CRIM1 affects thiopurine toxicity.

The present study proposes CRIM1 as a novel candidate pharmacogenetic gene for predicting thiopurine toxicity in pediatric ALL patients. The last-cycle 6-MP DIP for hematological toxicity measurements was used in estimating the independent and additive pharmacogenetic effects of CRIM1 over the well-known use of NUDT15 and TPMT. CRIM1 rs3821169 is a potentially deleterious (SIFT score $=0$ and CADD score $=25.3$ ) and very frequent variant in East Asian populations (minor allele frequency $=25 \%$ ), which increased the predictive power of the present analyses. As expected from the high allele frequency, the homozygous model improved the predictive accuracies for 6-MP intolerance. The heterozygous model demonstrated a moderate phenotypic effect. Recently, a novel association between $C Y P 2 A 7$ rs73032311 variant and 6-MP-induced leukopenia was reported in subjects with both WT NUDT15 and TPMT [17]. However, in our 240 ALL subjects with both WT NUDT15 and TPMT, the association signal of this variant was not replicated ( $p=0.891$ in age- and sex-adjusted multivariate linear regression analysis of the DIP model). None of the homozygote carriers exhibited DIP $<25 \%$ and showed slightly lower DIP $(61.51 \pm 13.9, n=6)$ compared to the heterozygote- $(68.83 \pm 30.3, n=56)$ and non-carriers $(66.08 \pm 30.7, n=178)$. It is suggested that CYP2A7 rs73032311 may have mild-to-moderate phenotypic effects on 6-MP intolerance only without sufficient clinical utility.

The allele frequency of CRIM1 rs3821169 $(T=0.255)$ is higher in East Asians than in other racial groups (global $=0.066, \quad$ Africans $=0.001$, Europeans $=0.009$, South Asians $=0.05$, and Americans $=0.02$; Phase 3 of the 1000 Genomes Project [13]). The homozygous carriers of this variant are identified only in the East Asian population $(T=0.071)$. This high interethnic variability might at least partly explain why rs3821169 has not yet been discovered as a biomarker for thiopurine toxicity. The current research bias toward Europeans [18] might have resulted in the statistical power being insufficient for this variant. The inclusion of a large (East Asian) Korean sample treated with 6-MP maintenance therapy $(n=320)$ in the present study allowed us to control the strong and well-known influences of NUDT15 and TPMT 
by defining the set of both-WT subjects for discovering further biomarkers.

The high interethnic variability of the pharmacogenetic variant is notable. The NUDT15 rs116855232 variant that was also very recently discovered to be a strong determinant of thiopurine toxicity in a Korean population [19] shows a much higher allele frequency in East Asians $(T=0.095)$ than in other ethnic groups (global $=0.040$, Africans $=0.001$, Europeans $=0.002$, South Asians $=0.07$, and Americans $=0.04$; Phase 3 of the 1000 Genomes Project [13]). In this study, one rare variant (rs780144127), to which the star allele has not yet been designated, was identified using whole exome sequencing (Additional file 1: Figure S9). The functional effect of this variant on thiopurine toxicity should further be demonstrated, as described in the previous works $[20,21]$. Unlike disease-causing genes, pharmacogenes by definition do not exhibit a phenotype unless exposed to the counterpart drug. The lack of overt disadvantageous phenotypes of these pharmacogenes might have permitted high interethnic variability and/or diversity under diverse evolutionary selection pressures in different surroundings.

\section{Conclusions}

In summary, CRIM1 is a gene associated with 6-MPinduced hematological toxicity. The evidence provided by this study was limited by the insufficient number of samples for the genome-wide significance and the lack of ethnic diversity. Further studies are needed to elucidate the role of CRIM1 in 6-MP metabolism.

\section{Supplementary information}

Supplementary information accompanies this paper at https://doi. org/10.1186/s12967-020-02416-7.

Additional file 1: Table S1. Evaluation of 12 candidate variants from the discovery cohort $(N=188)$ by using the replication cohort $(N=52)$ for both NUDT15 and TPMT wild-type subjects. Table S2. Evaluation of frequency distributions of CRIM1 rs3821169 genotypes across different cutoffs of the last-cycle 6-mercaptopurine dose intensity percentage tolerated by pediatric acute lymphoblastic leukemia subjects. Figure S1. Improvement of prediction accuracy of GVB ${ }^{\text {NUDT15,TPMT }}$ for thiopurine toxicity after controlling for homozygote carriers of CRIM1 rs3821169. Figure S2. Prediction accuracies of GVB ${ }^{\text {NUDT15,CRIM1 }}$ and GVB ${ }^{\text {TPMT,CRIM1 }}$ for thiopurine toxicity in pediatric ALL subjects. Figure S3. Prediction accuracies of GVB $^{\text {NUDT15,TPMT,CRIM1 }}$ for thiopurine toxicity in pediatric ALL subjects. Figure S4. Prediction accuracy of GVB ${ }^{C R I M 1}$ for thiopurine toxicity in pediatric $A L L$ subjects. Figure S5. Prediction accuracy of GVB ${ }^{N U D T 15}$ for thiopurine toxicity in pediatric ALL subjects. Figure S6. Prediction accuracy of GVB ${ }^{\text {TPMT }}$ for thiopurine toxicity in pediatric ALL subjects. Figure S7. Youden's index to find the optimal thresholds for GVB ${ }^{\text {NUDT15,TPMT }}$ and GVB NUDT15,TPMT,CRIM1 Figure S8. Comparison of CRIM1 mRNA expression levels of rs3821169 carriers and noncarriers in hematopoietic and lymphoid tissue. Figure S9. Results of Sanger sequencing for the two NUDT15 variants identified via whole exome sequencing.

\section{Abbreviations}

6-MP: 6-Mercaptopurine; ALL: Acute lymphoblastic leukemia; WES: Wholeexome sequencing; CPIC: Clinical Pharmacogenetics Implementation Consortium; WT: Wild type; DIP: Dose intensity percentage; SNUH: Seoul National University Hospital; AMC: Asan Medical Center; SMC: Samsung Medical Center; NM: Normal metabolizer; GVB: Gene-wise variant burden; SIFT: Sorting intolerant from tolerant; CADD: Combined annotation-dependent depletion; ROC: Receiver operating characteristic; AUC: Area under the ROC curve; CATT : Cochran-Armitage trend test; CRIM1: Cysteine Rich Transmembrane BMP Regulator 1; BMP: Bone morphogenetic protein.

\section{Acknowledgements}

Not applicable.

\section{Authors' contributions}

YP, HK, HJ and JHK designed the model and the framework. HJK, JYC, YM, KHY, $\mathrm{HJ}$ and HJK collected samples and clinical data. BJM and MES carried out the experiments. YP, HS, and SY analyzed the data and carried out the implementation of the idea. YP performed the calculations. YP and JHK wrote the manuscript. JHK and $\mathrm{HJ}$ conceived the study and were in charge of its overall direction and planning. All authors read and approved the final manuscript.

\section{Funding}

This research was supported by Grants from the Ministry of Food and Drug Safety in 2019 (No. 16183MFDS541) and the Korean Health Technology R\&D Project by Ministry of Health and Welfare in the Republic of Korea (No. HI18(2386).

\section{Availability of data and materials}

All data generated or analyzed during this study are included in this article. If any additional information is required, it may be obtained by request from the corresponding author.

\section{Ethics approval and consent to participate}

Informed written consent was obtained from all subjects, and the study was approved by the ethics committees of Asan Medical Center, Seoul National University Hospital, and Samsung Medical Center.

\section{Consent for publication}

Not applicable.

\section{Competing interests}

The authors declare that they have no competing interests.

\section{Author details}

${ }^{1}$ Division of Biomedical Informatics, Seoul National University Biomedical Informatics (SNUBI), Seoul National University College of Medicine, 101 Daehak-ro, Jongno-gu, Seoul 03080, South Korea. ${ }^{2}$ Department of Pediatrics, Asan Medical Center Children's Hospital, University of Ulsan College of Medicine, 88, Olympic-ro 43-gil, Songpa-gu, Seoul 05505, South Korea. ${ }^{3}$ Princess Margaret Cancer Centre, University Health Network, Toronto, ON M5G 1L7, Canada. ${ }^{4}$ Department of Pediatrics, Seoul National University College of Medicine, Seoul 03080, South Korea. ${ }^{5}$ Seoul National University Cancer Research Institute, Seoul, South Korea. ${ }^{6}$ Department of Pediatrics, Seoul National University Bundang Hospital, Seoul, South Korea. ${ }^{7}$ Department of Pediatrics, Samsung Medical Center, Sungkyunkwan University School of Medicine, Seoul, South Korea. ${ }^{8}$ Center for Precision Medicine, Seoul National University Hospital, Seoul 03080, South Korea.

Received: 13 January 2020 Accepted: 15 June 2020 Published online: 01 July 2020

\section{References}

1. Schaeffeler E, Jaeger SU, Klumpp V, Yang JJ, Igel S, Hinze L, et al. Impact of NUDT15 genetics on severe thiopurine-related hematotoxicity in patients with European ancestry. Genet Med. 2019;21(9):2145-50. 
2. Relling MV, Klein TE. CPIC: clinical pharmacogenetics implementation consortium of the Pharmacogenomics Research Network. Clin Pharmacol Ther. 2011;89(3):464-7.

3. Relling MV, Gardner EE, Sandborn WJ, Schmiegelow K, Pui CH, Yee SW, et al. Clinical pharmacogenetics implementation consortium guidelines for thiopurine methyltransferase genotype and thiopurine dosing: 2013 update. Clin Pharmacol Ther. 2013;93(4):324-5.

4. Relling MV, Schwab M, Whirl-Carrillo M, Suarez-Kurtz G, Pui CH, Stein CM, et al. Clinical pharmacogenetics implementation consortium guideline for thiopurine dosing based on TPMT and NUDT15 genotypes: 2018 update. Clin Pharmacol Ther. 2019;105(5):1095-105.

5. Yang JJ, Landier W, Yang W, Liu C, Hageman L, Cheng C, et al. Inherited NUDT15 variant is a genetic determinant of mercaptopurine intolerance in children with acute lymphoblastic leukemia. J Clin Oncol. 2015;33(11):1235-42.

6. Kim H, Seo H, Park Y, Min BJ, Seo ME, Park KD, et al. APEX1 polymorphism and mercaptopurine-related early onset neutropenia in pediatric acute lymphoblastic leukemia. Cancer Res Treat. 2018;50(3):823-34.

7. Park Y, Kim H, Choi JY, Yun S, Min BJ, Seo ME, et al. Star allele-based haplotyping versus gene-wise variant burden scoring for predicting 6-mercaptopurine intolerance in pediatric acute lymphoblastic leukemia patients. Front Pharmacol. 2019;10:654.

8. Cingolani P, Platts A, le Wang L, Coon M, Nguyen T, Wang L, et al. A program for annotating and predicting the effects of single nucleotide polymorphisms, SnpEff: SNPs in the genome of Drosophila melanogaster strain w1118; iso-2; iso-3. Fly. 2012;6(2):80-92.

9. Ng PC, Henikoff S. SIFT: predicting amino acid changes that affect protein function. Nucleic Acids Res. 2003;31(13):3812-4.

10. Kircher M, Witten DM, Jain P, O'Roak BJ, Cooper GM, Shendure J. A general framework for estimating the relative pathogenicity of human genetic variants. Nat Genet. 2014;46(3):310-5.

11. Lee KH, Baik SY, Lee SY, Park CH, Park PJ, Kim JH. Genome sequence variability predicts drug precautions and withdrawals from the market. PLoS ONE. 2016;11(9):e0162135.

12. Seo H, Kwon EJ, You YA, Park Y, Min BJ, Yoo K, et al. Deleterious genetic variants in ciliopathy genes increase risk of ritodrine-induced cardiac and pulmonary side effects. BMC Med Genomics. 2018;11(1):4.
13. Genomes Project C, Auton A, Brooks LD, Durbin RM, Garrison EP, Kang $\mathrm{HM}$, et al. A global reference for human genetic variation. Nature. 2015:526(7571):68-74.

14. Prenkert M, Uggla B, Tidefelt U, Strid H. CRIM1 is expressed at higher levels in drug-resistant than in drug-sensitive myeloid leukemia HL60 cells. Anticancer Res. 2010;30(10):4157-61.

15. Ziliak D, Gamazon ER, Lacroix B, Kyung Im H, Wen Y, Huang RS. Genetic variation that predicts platinum sensitivity reveals the role of miR-193 $\mathrm{b}^{*}$ in chemotherapeutic susceptibility. Mol Cancer Ther. 2012;11(9):2054-61.

16. Iorio F, Knijnenburg TA, Vis DJ, Bignell GR, Menden MP, Schubert M, et al. A landscape of pharmacogenomic interactions in cancer. Cell. 2016;166(3):740-54

17. Cao M, Yin D, Qin Y, Liao F, Su Y, Xia X, et al. Screening of novel pharmacogenetic candidates for mercaptopurine-induced toxicity in patients with acute lymphoblastic leukemia. Front Pharmacol. 2020;11:267.

18. Sirugo G, Williams SM, Tishkoff SA. The missing diversity in human genetic studies. Cell. 2019;177(4):1080

19. Yang SK, Hong M, Baek J, Choi H, Zhao W, Jung Y, et al. A common missense variant in NUDT15 confers susceptibility to thiopurine-induced leukopenia. Nat Genet. 2014;46(9):1017-20.

20. Moriyama T, Yang YL, Nishii R, Ariffin H, Liu C, Lin TN, et al. Novel variants in NUDT15 and thiopurine intolerance in children with acute lymphoblastic leukemia from diverse ancestry. Blood. 2017;130(10):1209-12.

21. Zhu Y, Yin D, Su Y, Xia X, Moriyama T, Nishii R, et al. Combination of common and novel rare NUDT15 variants improves predictive sensitivity of thiopurine-induced leukopenia in children with acute lymphoblastic leukemia. Haematologica. 2018;103(7):e293-5.

\section{Publisher's Note}

Springer Nature remains neutral with regard to jurisdictional claims in published maps and institutional affiliations.
Ready to submit your research? Choose BMC and benefit from:

- fast, convenient online submission

- thorough peer review by experienced researchers in your field

- rapid publication on acceptance

- support for research data, including large and complex data types

- gold Open Access which fosters wider collaboration and increased citations

- maximum visibility for your research: over $100 \mathrm{M}$ website views per year

At BMC, research is always in progress.

Learn more biomedcentral.com/submissions 